EPJ Web of Conferences 113, 04018 (2016)

DOI: $10.1051 /$ epjconf/201611304018

(C) Owned by the authors, published by EDP Sciences, 2016

\title{
The contribution of the ${ }^{3} \vec{H} e(\vec{\gamma}, n) p p$ reaction to the GDH integrand below pion production threshold
}

\author{
G. Laskaris ${ }^{1,2, a}$ \\ ${ }^{1}$ Triangle Universities Nuclear Laboratory, Durham, North Carolina 27708, USA \\ ${ }^{2}$ Department of Physics, Duke University, Durham, North Carolina 27708, USA
}

\begin{abstract}
The first measurements of the three-body phototdisintegration of ${ }^{3} \mathrm{He}$ polarized parallel and anti-parallel to a circularly polarized $\gamma$-ray beam were carried out at the High Intensity $\gamma$-ray Source $(\mathrm{HI} \gamma \mathrm{S})$ facility located at Triangle Universities Nuclear Laboratory (TUNL). A high pressure ${ }^{3} \mathrm{He}$ target, polarized via spin-exchange optical pumping with alkali metals, was used in the experiments. The neutrons from the three-body photodisintegration were detected with sixteen $12.7 \mathrm{~cm}$ diameter liquid scintillator detectors. The spin-dependent cross sections and the contributions from the three-body photodisintegration to the ${ }^{3} \mathrm{He}$ Gerasimov-Drell-Hearn sum rule integrand were extracted and compared with state-of-the-art three-body calculations at the incident photon energies of $12.8,14.7$, and $16.5 \mathrm{MeV}$. The calculations, which include the Coulomb interaction are in good agreement with the results of the measurements at 12.8 and $14.7 \mathrm{MeV}$ but deviate from the results at $16.5 \mathrm{MeV}$.
\end{abstract}

\section{Introduction}

Sum rules involving the spin structure of the nucleon and nuclei offer an important opportunity to study QCD. Among spin sum rules, the Gerasimov-Drell-Hearn (GDH) sum rule [1] is particularly interesting. It connects long distance behavior of the nucleon (nucleus), i.e. static property, to the entire excitation spectrum. The GDH sum rule relates the energy-weighted difference of the spindependent total photo-absorption cross sections $\sigma^{P}$ (for target spin and beam helicity parallel) and $\sigma^{A}$ (for target spin and beam helicity anti-parallel) to the anomalous magnetic moment of the target nucleus/nucleon as follows:

$$
I^{G D H}=\int_{v_{t h r}}^{\infty}\left(\sigma^{P}-\sigma^{A}\right) \frac{d v}{v}=\frac{4 \pi^{2} \alpha}{M^{2}} \kappa^{2} I,
$$

where $v$ is the photon energy, $v_{t h r}$ is the pion production/photodisintegration threshold on the nucleon/nucleus, $\kappa$ is the anomalous magnetic moment, $M$ is the mass and $I$ is the spin of the nucleon or the nucleus. This sum rule is based on fundamental principles such as Lorentz and Gauge invariance, crossing symmetry, causality and unitarity, and an assumption of unsubtracted dispersion relation. A detailed derivation of the GDH sum rule based on the aforementioned principles can be found in Ref. [2].

\footnotetext{
${ }^{\text {a}}$ Currently at Stanford University, Stanford, CA 94305, USA, e-mail: laskaris @ stanford.edu
} 


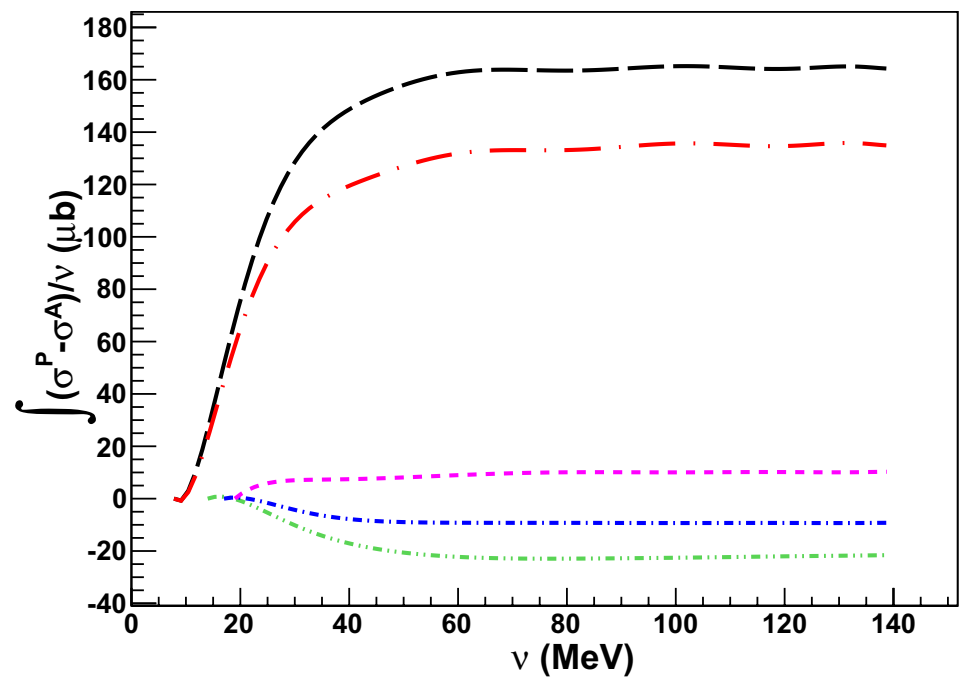

Figure 1. (Color online) Theoretical predictions of two- and three-body channel contributions to the ${ }^{3} \mathrm{He}$ GDH Integral below pion production threshold. The curves from top to bottom are: (i) long-dashed (black) curve: threebody channel contribution by Deltuva et al. (ii) long-dashed dotted (red) curve: three-body channel contribution by Skibiński et al. (iii) short-dashed (purple) curve: two-body channel contribution by Skibiński et al. (explicit MEC) (iv) short-dashed dotted (blue) curve: two-body channel contribution by Skibiński et al. (implicit MEC via Siegert theorem) (v) short-dashed double dotted (green) curve: two-body channel contribution by Deltuva et al.

The sum rule for ${ }^{3} \mathrm{He}$ can be written as the sum of two terms:

$$
496 \mu b=\int_{v_{t h r}}^{\infty} G D H_{3^{H e}}=\int_{v_{t h r}}^{v_{\pi}} G D H_{3} \mathrm{He}+\int_{v_{\pi}}^{\infty} G D H^{3} \mathrm{He}
$$

where the first term is from the two-body breakup threshold of ${ }^{3} \mathrm{He}(\sim 5.5 \mathrm{MeV})$ to the pion production threshold $(\sim 140 \mathrm{MeV})$ and the second term is for the energy region from the pion production threshold to infinity. The first term can be measured experimentally by carrying out double-polarized two-body and three-body photodisintegration experiments in this energy region and it can also be estimated based on the state-of the-art three-body calculations.

The state-of-the-art three-body calculations which can estimate the first part of the integral in Eq. (2) are performed mainly through the machinery of Faddeev [6] and Alt-Grassberger-Sandhas equations (AGS) [7], and have been carried out for both two-body and three-body photodisintegration of ${ }^{3} \mathrm{He}$ with double polarizations. These calculations $[8,9]$ use a variety of nucleon-nucleon $(\mathrm{NN})$ potentials like Argonne V18 (AV18) [10] or charge dependent (CD) Bonn [11, 12] and three-nucleon forces (3NFs) like Urbana IX (UIX) [13] or CD Bonn $+\Delta$ [8], with the latter yielding an effective $3 \mathrm{NF}$ through the $\Delta$-isobar excitation. The calculations by Deltuva et al. are based on AGS equations and employ the CD Bonn $+\Delta[8]$ with the corresponding single-baryon and meson-exchange electromagnetic currents plus relativistic single-nucleon charge corrections [8]. The proton-proton Coulomb force is included using the method of screening and renormalization [8]. Skibiński et al. solve the Faddeev equations by using the AV18 potential and the UIX 3NF accounting for single-nucleon currents 
and the two most important meson-exchange electromagnetic currents, the seagull and pion-in-flight terms [9].

Figure 1 shows the GDH integral for the two- and three-body photodisintegration of ${ }^{3} \mathrm{He}$ below pion production threshold. The short-dashed double dotted (green) and the long-dashed (black) curves are the estimations of the GDH integral for the two- and three-body photodisintegration from Deltuva et al. Their sum amounts to $142 \mu \mathrm{b}$. The short-dashed (purple) and short-dashed dotted (blue) curves are two different estimations by Skibiński et al. of the two-body channel contribution to the GDH integral taking into account explicitly or implicitly the MEC via the Siegert theorem. The longdashed dotted curve is the estimation of the three-body contribution to the GDH integral coming from the same group. The maximum estimation of the GDH integral below pion threshold according to Skibiński et al. is $145 \mu \mathrm{b}$. Both theoretical calculations predict that the contribution to the GDH sum rule below pion production threshold is $140-150 \mu \mathrm{b}$ which is an important part of the overall GDH integral (496 $\mu \mathrm{b})$. This contribution lays mainly below the incident photon energy of $40 \mathrm{MeV}$. Therefore, a spin-dependent study of ${ }^{3} \vec{H} e(\vec{\gamma}, n) p p$ below $40 \mathrm{MeV}$ not only provides a stringent test of modern three-body calculations, but also serves as an important step towards an experimental test of the GDH sum rule on the ${ }^{3} \mathrm{He}$ nucleus.

\section{The Experiment}

The first experiments $[14,15]$ on the three-body photodisintegration of ${ }^{3} \mathrm{He}$ using a longitudinally polarized ${ }^{3} \mathrm{He}$ target and a circularly polarized $\gamma$-ray beam took place at the HI $\gamma \mathrm{S}$ facility [17] of TUNL at the incident photon energies of $12.8,14.7$ and 16.5 MeV. A nearly mono-energetic, 100\% circularly-polarized pulsed $\gamma$-ray beam was used. The beam was collimated using a $12 \mathrm{~mm}$ diameter collimator resulting in on-target intensities of $(0.7-2.0) \times 10^{8} \gamma / \mathrm{s}$ and an energy spread of $\Delta v / v \sim 3.0$ $5.0 \%$. The on-target intensity of the beam was determined using the well-known $\mathrm{d}(\gamma, \mathrm{n}) \mathrm{p}$ cross section [18] and two BC501A liquid scintillator neutron detectors mounted at a scattering angle of $90^{\circ}$ degrees downstream of the ${ }^{3} \mathrm{He}$ target.

Upstream of the flux monitor, the polarized $\gamma$-beam was incident on a polarized ${ }^{3} \mathrm{He}$ cell. A $\mathrm{N}_{2}$ reference cell was used for background subtraction. Details concerning their technical characteristics and the spin exchange optical pumping technique used to polarize the ${ }^{3} \mathrm{He}$ target can be found in Refs. [14, 15, 19-21]. The spin of the ${ }^{3} \mathrm{He}$ target was flipped every $15 \mathrm{mins}$ in order to extract the spin-dependent cross sections and the GDH integrand, $\left(\sigma^{P}-\sigma^{A}\right) / v$. The polarization was measured using the nuclear magnetic resonance-adiabatic fast passage [22] technique calibrated by electron paramagnetic resonance [23]. The latter can measure the absolute ${ }^{3} \mathrm{He}$ target polarization which was found to be between $33 \%$ and $45 \%$.

An array of sixteen liquid scintillator BC-501A counters was used to detect the neutrons from the ${ }^{3} \vec{H} e(\vec{\gamma}, n) p p$ reaction. The detectors were placed at the horizontal plane every $15^{\circ}$, symmetrically on each side of the beam, at laboratory scattering angles from $30^{\circ}$ to $165^{\circ}$. No detectors were placed at the laboratory angles $60^{\circ}$ and $120^{\circ}$ due to the proximity to a pair of Helmholtz coils which provided the holding field for the polarized ${ }^{3} \mathrm{He}$ target.

Three quantities were recorded for each event: the pulse height (PH), the time-of-flight (TOF) and the pulse shape discrimination (PSD) signals. Initially, a PH cut was applied at $0.162 \mathrm{MeVee}$ to set the detector efficiency. The correlations between the PSD, PH and TOF were utilized to extract the neutron events and to remove the $\gamma$-ray events and two-dimensional cuts were applied on these histograms. The same cuts were used for the data taken with the $\mathrm{N}_{2}$ reference cell to subtract the background contributions. The outgoing neutron energy was determined using the measured TOF of 
the neutrons assuming they were emitted from the center of the ${ }^{3} \mathrm{He}$ target cell. More details about this analysis can be found in Refs. [14, 15].

\section{Results and Discussion}

The spin-dependent double-, single-differential and total cross sections, and the contributions of the three-body photodisintegration of ${ }^{3} \mathrm{He}$ to the GDH integrand were obtained [14-16] and compared with the state-of the-art three-body calculations [8, 9] at all incident energies. Fig. 2 [16] shows the GDH integral results including the statistical and systematic uncertainties compared with the theoretical calculations. Although a very good agreement is observed between the measurements [14, 15] and the calculations based on Ref. [8] at 12.8 and $14.7 \mathrm{MeV}$, a difference between the measured GDH integrand and the calculations can be seen at the incident photon energy of $16.5 \mathrm{MeV}$. The measured GDH integrand at $16.5 \mathrm{MeV}$ is found to be slightly more than one standard deviation larger than the maximum calculated value based on Ref. [8].

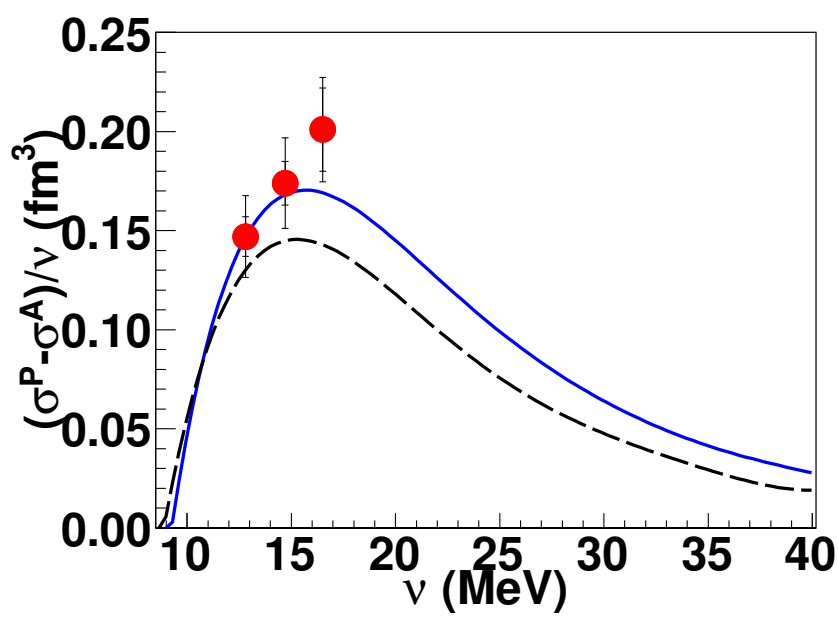

Figure 2. (Color online) The combined GDH integrand results as can be seen in Ref. [16] compared with the theoretical predictions of Ref. [8] (solid-blue curve) and Ref. [9] (dashed-black curve). The inner error bars of the data points represent the statistical uncertainties while the outer include both the statistical and systematic uncertainties added in quadrature.

To investigate whether the larger than expected GDH integrand value at $16.5 \mathrm{MeV}$ is due to statistics and to further quantify the three-body contribution to the GDH integral, measurements above 16.5 $\mathrm{MeV}$ for the three-body photodisintegration channel are necessary. These measurements combined with the recently acquired data from the two-body photodisintegration channel [15] will constrain the contribution to the GDH integral for ${ }^{3} \mathrm{He}$ below the pion-threshold.

\section{Acknowledgements}

The author wish to thank the members of the Medium Energy Physics group and the Capture group of Duke University, A. Deltuva, A.C. Fonseca, J. Golak, P. U. Sauer, R. Skibiński and H. Witała for providing theoretical calculations, Michael Souza of Princeton University for making the target cells, Gordon Cates' group from 
University of Virginia for providing the $\mathrm{Rb} / \mathrm{K}$ ampules and for Sol-Gel coating of the target cells, $\mathrm{T}$. Averett for filling the cell and the TUNL personnel for the technical support of this experiment. This work is supported by the U.S. Department of Energy under contract number DE-FG02-03ER41231 and Duke University.

\section{References}

[1] S.D. Drell and A.C. Hearn, Phys. Rev. Lett. 16, 908 (1966); S.B. Gerasimov, Yad. Fiz. 2, 598 (1965) (Sov. J. Nucl. Phys. 1, 430 (1966)).

[2] K. Helbing, Progress in Particle and Nuclear Physics 57, 405 (2006).

[3] J.L. Friar, B.F. Gibson, G.L. Payne, A.M. Bernstein, and T.E. Chupp, Phys. Rev. C 42, 2310 (1990).

[4] P. Aguar Bartolomé et al., Phys. Lett. B 723, 71 (2013).

[5] S. Costanza, Eur. Phys. J. A 50, 173 (2014).

[6] L.D. Faddeev, Zh. Eksp. Theor. Fiz. 391459 (1960); Sov. Phys. JETP 12, 1041 (1961).

[7] E.O. Alt, P. Grassberger, and W. Sandhas, Nucl. Phys. B2, 167 (1967).

[8] A. Deltuva, L.P. Yuan, J. Adam, A.C. Fonseca, P.U. Sauer, Phys. Rev. C 69, 034004 (2004); A. Deltuva, A.C. Fonseca, and P.U. Sauer, Phys. Rev. C 72, 054004 (2005); Ann. Rev. Nucl. Part. Sci. 58, 27 (2008); A. Deltuva, A.C. Fonseca, and P.U. Sauer, Phys. Rev. C 80, 064004 (2009).

[9] R. Skibiński, J. Golak, H. Witała, W. Glöckle, A. Nogga, and H. Kamada, Phys. Rev. C 72, 044002 (2005); R. Skibiński, J. Golak, H. Witała, W. Glöckle, H. Kamada and A. Nogga, Phys. Rev. C 67, 054002 (2003); R. Skibiński, J. Golak, H. Kamada, H. Witała, W. Glöckle and A. Nogga, Phys. Rev. C 67, 054001 (2003).

[10] R. B Wiringa, V. G. J. Stoks, R. Schiavilla. Phys. Rev. C 51, 38 (1995).

[11] R. Machleidt and K. Holinde and Ch. Elster, Phys. Rep. 149, 1 (1987).

[12] R. Machleidt, Phys. Rev. C 63, 024001 (2001).

[13] J. Carlson, V.R. Pandharipande, and R.B. Wiringa, Nucl. Phys. A 401, 59 (1983).

[14] G. Laskaris et al., Phys. Rev. Lett. 110, 202501 (2013); Phys. Rev. C 89, 024002 (2014).

[15] G. Laskaris, Ph.D. thesis, Duke University (2015).

[16] G. Laskaris et al., Phys. Lett. B 750C, 547 (2015);

[17] H.R. Weller M.W. Ahmed, H. Gao, W. Tornow, Y.K. Wu, M. Gai, and R. Miskimen, Prog. Part. Nucl. Phys. 62, 257 (2008).

[18] D.M. Skopik, Y.M. Shin, M.C. Phenneger, and J.J. Murphy II, Phys. Rev. C 9, 531 (1974); Y. Birenbaum, S. Kahane, and R. Moreh, Phys. Rev. C 32, 1825 (1985); R. Bernabei et al., Phys. Rev. Lett. 57, 1542 (1986); A. De Graeve et al., Phys. Rev. C 45, 860 (1992).

[19] W. Happer Rev. Mod. Phys. 42, 169 (1972).

[20] K. Kramer, X. Zong, R. Lu, D. Dutta, H. Gao, X. Qian, Q. Ye, X. Zhu, T. Averett, and S. Fuchs, Nucl. Instrum. Methods Phys. Res., Sect. A 582, 318 (2007).

[21] Q. Ye, G. Laskaris, W. Chen, H. Gao, W. Zheng, X. Zong, T. Averett, G.D. Cates, and W.A. Tobias, Eur. Phys. J. A 44, 55 (2010).

[22] W. Lorenzon, T.R. Gentile, H. Gao, R.D. McKeown, Phys. Rev. A 47, 468 (1993).

[23] M.V. Romalis and G.D. Cates, Phys. Rev. A 58, 3004 (1998), and the references therein. 